Research Article

\title{
Cloud Computing-Based Medical Health Monitoring IoT System Design
}

\author{
Shihua Cao $\mathbb{D}^{1},{ }^{1}$ Xin Lin, ${ }^{2}$ Keyong Hu $\left(\mathbb{D},{ }^{1}\right.$ Lidong Wang $\mathbb{D}^{1},{ }^{1}$ Wenjuan Li $\left(\mathbb{D},{ }^{1}\right.$ Mengxin Wang, ${ }^{3}$ \\ and Yuchao Le $^{3}$ \\ ${ }^{1}$ Department of Nursing, Hangzhou Normal University Qianjiang College, Hangzhou, Zhejiang 310036, China \\ ${ }^{2}$ Bengbu 3rd Hospital, Bengbu, Anhui 233000, China \\ ${ }^{3}$ School of Medicine, Hangzhou Normal University, Hangzhou, Zhejiang 310012, China \\ Correspondence should be addressed to Shihua Cao; csh@hznu.edu.cn
}

Received 25 May 2021; Revised 12 June 2021; Accepted 25 June 2021; Published 8 July 2021

Academic Editor: Sang-Bing Tsai

Copyright (c) 2021 Shihua Cao et al. This is an open access article distributed under the Creative Commons Attribution License, which permits unrestricted use, distribution, and reproduction in any medium, provided the original work is properly cited.

With the continuous improvement of the national medical system, health monitoring combined with cloud computing and Internet of Things has become a concern. This study mainly discusses the design of the medical health monitoring IoT system based on cloud computing. From the user to the health service provider, there are three devices: sensor terminal, gateway terminal, and service platform. The sensor terminal is used to measure physiological indicators, such as blood pressure, electrocardiogram, blood oxygen saturation, heart rate, and other physiological indicators; the gateway terminal is used to link the sensor terminal to receive physiological indicators and forward them to the business platform; the gateway is also used to receive health information and other instructions issued by the server. In the community service mode, users can be divided into groups according to the community and region, and the corresponding service doctors and agent customer service personnel (nurses) can be assigned. Users can collect personal physiological indicators at home or outside through the medical terminal. These indicators and information are transmitted to the background health platform system through the mobile GSM-TD communication network. Users can also view their own historical health records and opinions of health consultants through the web/WAP website. Through the integration ability of the health cloud platform, relying on the interconnection with HIS, LIS, and other information systems of professional medical institutions, we jointly operate special value-added services, such as appointment registration, maternal and child healthcare, and medical communication (doctor-patient interaction), so that users can enjoy the remote service and guidance of professional medical institutions by subscribing to health value-added services. The CPU utilization rate is $40 \%$, the internal utilization rate is $7.44 \mathrm{G}$, the memory utilization rate is $11.8 \%$, and the network bandwidth is $591.87 \mathrm{M}$. During the whole test process, the indicators are stable, and there are no restart, crash, and other phenomena, so the system performance meets the design requirements.

\section{Introduction}

With the inevitable aging of population and the increasing attention of people to health problems, the proportion of medical expenses in China's GDP has gradually increased. Therefore, driven by the trillions of market value in the medical field, it began to combine big data technology to apply to the clinical management and research of medicine.

In the process of continuous informatization in medical field, to solve the challenge of massive medical data, especially the security storage problem, major companies have launched some targeted solutions for safe storage. Therefore, it is of practical significance to carry out the research on the security storage of medical data in cloud computing environment.

The Internet of Things (IoT) is a new paradigm. Mishra A K believes it is important to understand the attacker's behavior and predict what it may take while designing a defense mechanism. He has carried out a comprehensive feature analysis of Sybil attacks in the Internet of Things. Compromise stage is modeled as automaton, and attacker state is transformed into Markov chain model. He also 
proposes a heuristic method for attackers to use the selection criteria to destroy nodes. In the deployment stage, he proposes a $\mathrm{k}$-means clustering algorithm to group the damaged identity and deploy Sybil nodes for the corresponding identity. Although he used age replacement strategies to model the process of replacing Sybil identity over time or when it was detected during the start-up phase, his research process was not practical [1]. Shifa A believes that physical objects can be connected to each other through the Internet and connected to users and can share information between devices and systems. In the Internet of Things environment, he deployed intelligent devices to monitor, control, and analyze business, personal, and social activities. He reviewed the IoT layered architecture and proposed security challenges to build an IoT environment. Although he then proposed a solution to retain the privacy of multimedia data in IoT environment of its perception layer, his research is not novel enough [2]. The Internet of Things gateway is the key intermediary between many intelligent things and their corresponding cloud network servers. Chen $\mathrm{C} \mathrm{H}$ thinks that the typical conventional gateway system is based on advanced embedded microcontroller (MCU). He proposes an innovative multi-MCU system framework, which combines hardware bridge based on field programmable gate array and multiple extensible MCU to realize edge gateway of intelligent sensor fieldbus. Although he proposed the concept of edge gateway, he could not provide IoT functions with single MCU solution on system scalability, computing efficiency, and communication efficiency, such as big data collection, management, real-time communication, scalable peripheral devices, and various other services $[3,4]$. Centenaro $M$ introduces the architecture of the next-generation mobile technology on the Internet of Things based on cloud and its unique security and privacy requirements. It determines the inappropriate points of most existing work and solves the challenge of secure packet forwarding and effective privacy protection authentication by providing solutions. Although he proposed a new high-efficiency privacy protection data aggregation without public key homomorphism encryption, his research process lacks data [5].

With the continuous improvement of the national medical system, health monitoring combined with cloud computing and Internet of Things has become a concern. This study mainly discusses the design of medical health monitoring IoT system based on cloud computing. From the user to the health service provider, there are three devices: sensor terminal, gateway terminal, and service platform. The sensor terminal is used to measure physiological indicators, such as blood pressure, electrocardiogram, blood oxygen saturation, heart rate, and other physiological indicators; the gateway terminal is used to link the sensor terminal to receive physiological indicators and forward them to the business platform; the gateway is also used to receive health information and other instructions issued by the server. In the community service mode, users can be divided into groups according to the community and region, and the corresponding service doctors and agent customer service personnel (nurses) can be assigned. Users can collect personal physiological indicators at home or outside through the medical terminal. These indicators and information are transmitted to the background health platform system through the mobile GSM-TD communication network. Users can also view their own historical health records and opinions of health consultants through the web/WAP website. Through the integration ability of health cloud platform, relying on the interconnection, we jointly operate special value-added services, such as appointment registration, maternal and child healthcare, and medical communication (doctor-patient interaction), so that users can enjoy the remote service and guidance of professional medical institutions by subscribing to health value-added services. During the whole test process, the indicators are stable, and there are no restart, crash, and other phenomena, so the system performance meets the design requirements.

\section{Medical Health Monitoring IoT System}

2.1. Cloud Computing Platform. The use of the chronic disease big data secure storage system in the cloud computing environment can greatly reduce the storage cost of chronic disease data, borrow the powerful computing power of cloud computing to access the stored data and enable the designed audit strategy to be completed quickly, and then improve storage security. The designed access control rules can limit the user's authority and then reduce unnecessary operation errors and malicious attacks; through the audit technology, the stored chronic disease data is checked for security, so as to achieve the purpose of chronic disease data privacy protection $[6,7]$. The spatial range that the perception layer $V(\varepsilon, T)$ can recognize is as follows:

$$
F_{B}(\varepsilon, T)=\sqrt{\frac{1-k(\varepsilon, T)}{[1+k(\varepsilon, T)][1-2 k(\varepsilon, T)] \rho(\varepsilon, T)}} .
$$

Among them, $T$ is the space temperature [8]. The energy consumed in each round of monitoring data $S$ is as follows:

$$
S=\left(\frac{N}{k-1}\right) l E_{\text {elec }}^{R x}+\left(\frac{N}{k l E_{D A}}\right)+\left(l E_{\text {elec }}^{T x}+l \varepsilon_{\text {amp }} d_{t o B S}^{n}\right) .
$$

Among them, $l$ represents the length of the data packet [9]. If there is an MF attack on the authentication process of message $m$ in the DB protocol, the success probability $P_{C}$ is

$$
P_{C}=P_{c^{i}}^{n}+\lambda \sum_{k=0}^{m-1}\left(\begin{array}{l}
n \\
k
\end{array}\right) P_{c^{i}}^{k}\left(1-P_{c^{i}}\right)^{n-k}\left(\frac{1}{C}\right)^{n-k} .
$$

Among them, Crepresents the size of the candidate space of response bits in the fast exchange phase $[10,11]$.

$$
H X(P \| Q)=\frac{1}{2}\left(D_{K L}(P \| M)\right)+D_{K L}(Q \| M) .
$$

Among them, 


$$
M=\frac{1}{3}(P+Q) .
$$

Connectivity is the most basic building block of the IoT paradigm [12].

$$
L(P \| Q)=\sum_{x \in \psi} P(x) \log \frac{P(x)}{Q(x)} .
$$

Among them, $Q(x)$ is the probability distribution [13].

$$
T_{L}=\int p(z \mid p) T(z) d z .
$$

If $T_{p}=p$ is estimated on average, it is called unbiased estimation. For unbiased estimation, there are [14]

$$
Q=\int p\left(z_{1} \mid p\right) \cdots p\left(z_{m} \mid p\right) \Delta T d z_{1} \cdots d z_{m} .
$$

For the partial derivative of $p$ in the above formula, we get

$$
Q=\sum_{i=1}^{m} \int p\left(z_{1} \mid p\right) \cdots p\left(z_{m} \mid p\right) \frac{1}{p\left(z_{1} \mid p\right)} \frac{\partial p\left(z_{1} \mid p\right)}{\partial p} \Delta T d z_{1} .
$$

Because $p$ is dependent on $T_{p}$ instead of $T$, the above formula can be converted to $[15,16]$

$$
Q=\int p\left(z_{1} \mid p\right) \cdots p\left(z_{1} \mid p\right) \frac{1}{p\left(z_{1} \mid p\right)}\left(\sum_{i=1}^{m} \frac{\partial \ln p\left(z_{1} \mid p\right)}{\partial p}\right) \Delta T d z_{1} .
$$

Using Cauchy-Schwarz inequality [17],

$$
\left[\int f(x) g(x) d x\right]^{2} \approx \int f(x)^{2} d x \int g(x)^{2} d x .
$$

Available is

$$
\int T\left(z_{m} \mid p\right)\left(\sum_{i=1}^{m} \frac{\partial \ln T\left(Z_{i} \mid T\right)}{\partial T}\right)^{2} d Q_{1} \geq\left|\frac{d T_{p}}{d T}\right|^{2} .
$$

2.2. Internet of Things. Internet of Things medical care includes core functions such as medical and health data collection, identification, positioning, tracking management, etc. It uses intelligent network and communication technology to connect individuals, medical staff, and various sensors, and the application space is very broad. It will introduce the IoT technology of sensor nodes based on short-range communication technology and home wireless IoT technology $[18,19]$. The perfect combination of the Internet of Things and remote health monitoring technology allows people to monitor their physical condition and exercise status at any time. First of all, it is beneficial to patients to obtain the best disease prevention and treatment results and the country's reforms to improve medical and health public services and security capabilities. The ideas are consistent [20]. Introduce Fisher's information $Q(p)$, and get

$$
\begin{aligned}
m Q(p)(\Delta T)^{2} & \geq\left|\frac{d T_{p}}{d p}\right|^{2}, \\
H & =\frac{1}{\|w\|}\left(w^{T} x_{0}+b\right) .
\end{aligned}
$$

Then normalize it to make the linearly separable training set $\left(x_{i}, y_{i}\right)$ :

$$
R=y_{i}\left(w^{T} x_{i}+b\right)-1
$$

At this time, the distance from the dividing line to both sides is equal to $2 / \| w^{2}$ :

$$
F=\arg \max \left\{\frac{1}{\left\|w^{2}\right\|} \min \left[y_{i}\left(w_{n}^{T} x_{i}+b\right)\right]\right\} .
$$

2.3. Medical Monitoring. Medical health monitoring is the core of this research $[21,22]$.

Personal information management: record different individual situations of each user, including the user's basic personal information (such as gender, age, height, weight, etc.), personal health history (including family medical history, medication history, smoking, and other lifestyle habits), terminal collection records, etc.; record and store the user's physical indicators and other data. When a complete personal health file is built, the user's health can be monitored for a long time, and the health status can be evaluated in combination with the long-term monitoring $[23,24]$.

Summary of medical data: transform IT applications from commonly used and quantifiable analysis methods in the medical field to realize automatic or semiautomatic data analysis. The content includes daily analysis trend chart (an analysis chart every day, showing the occurrence time of abnormal indicators of the day in time), weekly analysis trend chart (an analysis chart every week, showing the occurrence time of abnormal indicators in a week by day), month analysis trend graph (an analysis graph per month, showing the occurrence time points of abnormal indicators in a day by day), and statistical graphs of the occurrence time of abnormal indicators (summarizing the occurrence time points of indicator abnormalities within a period of time and using it to judge when the onset period is) $[25,26]$.

For example, for a hypertensive patient, after measuring his blood pressure, these data can be uploaded to the monitoring system via the Internet, and the system's medical standard rules can be invoked for calculation and reasoning, and a set of targeted data can be given. For health guidance programs or promotion suggestions $[27,28]$, the medical health monitoring process based on cloud computing and Internet of Things technology is shown in Figure 1.

Although the objective function is clearly expressed. Here we use a series of mathematical operations such as the Lagrangian multiplier method to finally obtain the objective function: 


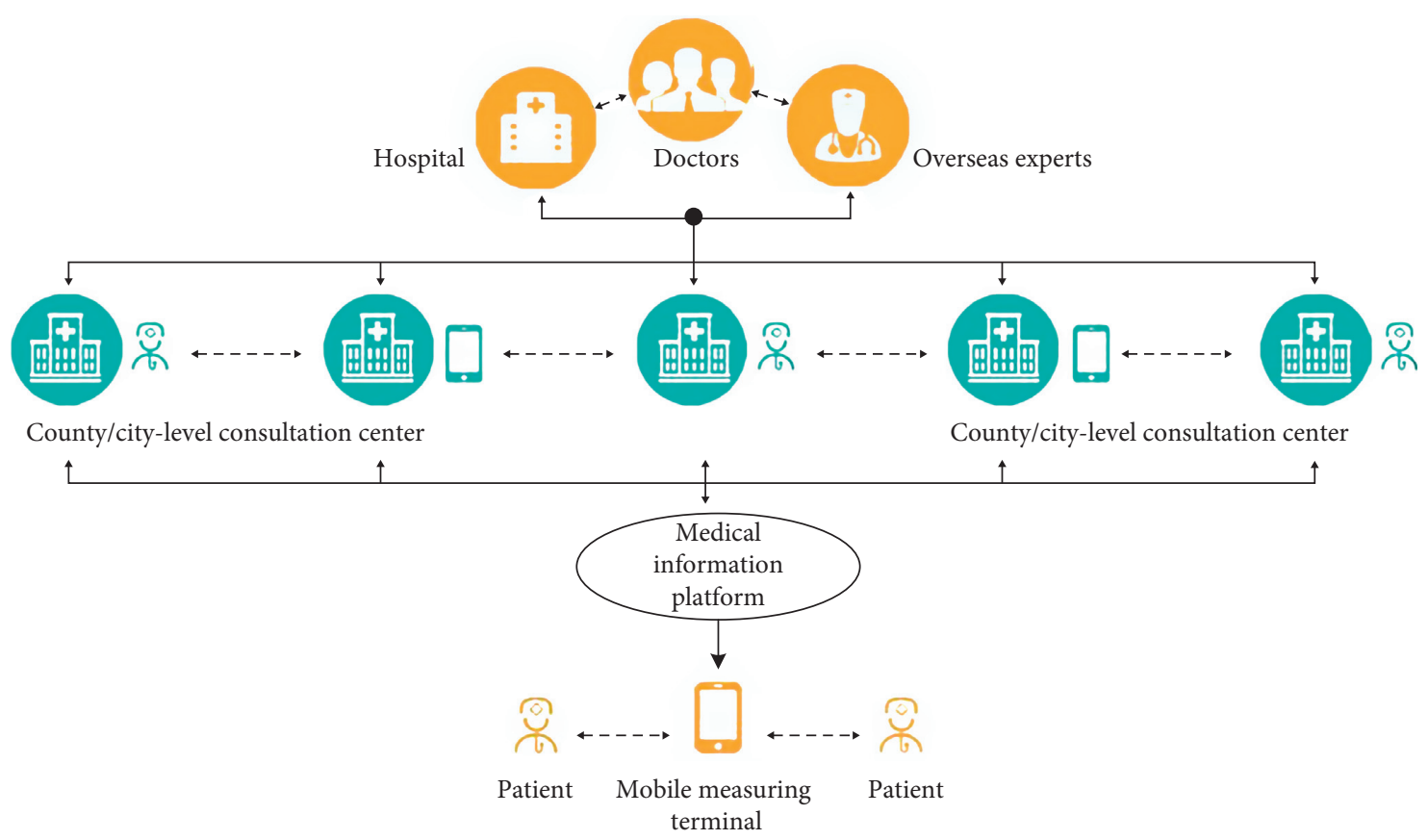

FIGURE 1: Medical health monitoring process based on cloud computing and Internet of Things technology (some pictures are from Baidu).

$$
Y(S)=\sum_{n=1}^{N} S_{n}-\frac{1}{2} \sum_{n=1}^{N} \sum_{m=1}^{N} S_{n} y_{m} x_{n}^{T} x_{m} .
$$

Among them,

$$
\sum_{n=1}^{N} S_{n} y_{n}=0
$$

Take $x_{i}$ as a sample of training data set $T\left(r_{x}\right)[24,29]$.

$$
T\left(f_{i}, f_{j}\right)=\iint p\left(f_{i}, f_{j}\right) \log \frac{p\left(f_{i}, f_{j}\right)}{p\left(f_{i}\right) p\left(f_{j}\right)} d f_{i} d f_{j} .
$$

Here, $p\left(f_{i}\right)$ is the probability distribution of feature $f_{i}$ [30]. Then, the least mean square estimate of the state variable $X$ at time $i$ is

$$
X(U, V)=\sum_{i=1}^{c} \sum_{j=1}^{n} \mu_{i j}^{m}\left\|x_{j}-v_{i}\right\|^{2}
$$

\section{Cloud Computing Medical Health Monitoring IoT System Experiment}

3.1. Overall Functions of the Medical and Health Service System. The mobile medical health service system can collect blood pressure, blood glucose, blood oxygen, electrocardiogram, heart rate, body temperature, breathing rate, and other physiological indicators through health measurement terminals and then transfer the physiological indicators to the business platform system through the TDSCDMAGSM mobile communication network. The indicators are stored and presented, and physiological indicators are preanalyzed, and historical trend is analyzed at the same time. Users can also customize related services [31].

The mobile medical health service system consists of three parts: cloud platform, gateway terminal, and sensor terminal. The cloud platform carries business access and provides powerful storage, analysis, reminder, and other service functions and management functions; the sensor terminal is responsible for the measurement and acquisition of health indicators; the gateway terminal is responsible for gathering and uploading the indicator data measured by the sensor to the platform and accepting all kinds of information issued by the cloud platform.

\subsection{Health Service Business}

3.2.1. Basic Healthcare Services. The basic healthcare service means that users can collect personal physiological indicators through medical terminals at home or outside. These indicators and information are connected to the home information machine or mobile phone through the mobile GSM-TD communication network (that is, the terminal is connected to the home information machine or mobile phone through the short-range Bluetooth mode). Use it as a transmission gateway to transmit data to the backend health platform system. For the trend of single information or multiple information, the computer expert system will make a preliminary analysis and submit the analysis results to the corresponding health consultant or professional doctor. They can combine the user's health file information to give targeted health interventions or recommendations for medical treatment. The doctor's suggestion will notify the user through SMS or MMS. In addition, users can also check their own historical health records and the opinions of health consultants in detail through the WEB/WAP website, 
fully understand the development of personal chronic diseases, and take early measures to control the development of the disease.

3.2.2. Health Value-Added Services. In addition to basic health protection services, the health service platform can also provide health value-added services in two ways: one is that after users use basic health protection, if they find that they belong to a certain disease or characteristic, they can directly subscribe to existing ones through mobile 12580 standardized businesses such as health consultants, chronic disease knowledge, etc., to obtain healthcare knowledge and key points for disease prevention and treatment; the second method is to rely on the integration of the health cloud platform to rely on the interconnection with professional medical institutions HIS, LIS, and other information systems, jointly operating special value-added services, such as appointment registration, maternal and child healthcare, medical information communication (doctor-patient interaction), etc., so that users can enjoy remote services and guidance from professional medical institutions by subscribing to health value-added services. It can also gradually operate products such as emergency patient education and medical equipment inquiry to expand new target markets and user groups.

There are three devices between users and health service providers: sensor terminals, gateway terminals, and business platforms. The sensor terminal is used to measure physiological indicators, such as blood pressure, electrocardiogram, blood oxygen saturation, heart rate, and other physiological indicators; the gateway terminal is used to link the sensor terminal to receive physiological indicators and forward them to the business platform; the gateway is also used to receive the health issued by the server information and other instructions. Sensors and gateways form a terminal system for mobile health services. There are two types of interfaces between the three types of devices: $T$ interface and $S$ interface. $T$ interface is the remote communication interface between the gateway and the platform server, including wire or wireless mode; $\mathrm{S}$ interface is the short distance communication interface between the gateway and the sensor, which is also divided into wired and wireless modes. The connection between the sensor terminal, the gateway terminal, and the service platform is shown in Figure 2.

3.3. Medical Institution Management Module. The system supports operation and management personnel to register new medical service organization information in the system, including basic information of the medical organization, department organization structure, doctor/nurse information, medical service information, and duty information.

The hospital refers to the network hospital, which is under the responsibility of the dean, and the hospital contains multiple departments. As a manager, you can set up various departments to deal with related diseases, for example, "cardiovascular department," which mainly deals with cardiovascular diseases. In order to manage users, users in the same department (cardiovascular department) can be grouped and managed. Grouping usually gathers users by geographic location to facilitate the conduct of centralized door-to-door visits by users in the group. According to calculations, a group roughly contains 200 users. When the user scale is larger, the division level can be introduced above the grouping to form a 4-level structure for better management.

As a doctor in the hospital, he will be able to decide that a certain user belongs to a certain group. You can also arrange which doctors in the undergraduate room serve different packages and which users to serve, define each group of a certain department of the hospital, and arrange which seats belong to a certain group. The dean can define the departments of the hospital based on the management content of the chief doctor.

In the on-duty management, the chief doctor can schedule and manage the doctors and nurses in the undergraduate room on the management portal and define the duty agents and doctors in time periods each week. If there is a temporary event conflict, you can modify it. The system maintains all scheduling information in the hospital. The chief doctor can view the scheduling information in the undergraduate room, and the dean can view the scheduling information of all departments in the hospital. When the system receives a call from a user, it needs to select one of the seats to be connected according to the duty information. The system administrator assigns permissions to different user positions on the management portal.

3.4. User Management Module. Market business operators can register and open accounts and configure and manage user profiles according to the user's disease type and selected package information. In the community service mode, users can be divided into groups according to communities and regions, and corresponding service doctors and agent customer service staff (nurses) can be assigned. The system can perform real-time diagnosis and treatment analysis services on the user's pathological data indicators reported through the medical terminal and feedback to the user after confirmation by the medical service personnel. Specifically, user management includes the following aspects.

3.4.1. User Registration Management. This function incorporates the user into the system and evaluates whether the user is suitable for the system's service objects. Registration management can be handled in hospitals and mobile business halls, and the system supports single user entry and batch import.

The information that needs to be provided by the user is basic personal information (name, gender, birth date, ID number, home address, and contact phone number), health information (height, weight, blood type, drug allergy, heart disease, diabetes, high blood pressure, and asthma), financial information (deductible account amount), guardian information (name, relationship, contact number, and address), types of diseases to be ordered, and personal recent physical examination form (heart disease-providing 


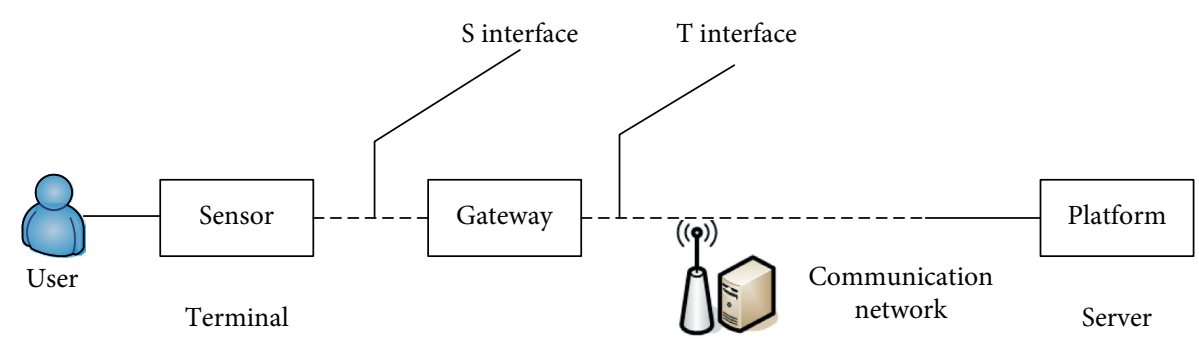

Figure 2: The connection between the sensor terminal, the gateway terminal, and the business platform.

electrocardiogram, hypertension-providing blood pressure physical examination form, blood sugar one to provide a biochemical physical examination form).

After passing the review, the chief doctor will give a suggested package and notify the user to accept the package. When the doctor in charge of the audit recognizes a patient with a serious condition, he should recommend going to the hospital directly, and the process is returned to the market staff for persuasion. Some patients can meet the requirements of remote health management but need higher-level services. At this time, they need to call back to the market staff for persuasion. Physiological information (electrocardiogram, blood pressure, and blood sugar) can be collected on-site when registering at a medical institution.

3.4.2. Package Acceptance. After confirming that the user meets the system's service target standards, the user can go to the mobile business hall or service providing medical institution to apply for a chronic disease management package. Package acceptance determines the order relationship and synchronizes to the BOSS. At the same time, it can notify the customer service staff, install terminal equipment on-site and train customers to use it, confirm that the equipment is available and the user's subscription service is available, and also confirm the correctness of the user's key information, including home address and guardian information.

3.4.3. User Detection Service. When the customer service installs the device, it needs to verify the correctness and availability of the user number, relative number, community number, etc., and notify the user to officially open the business. It also needs to include medical order management, medical order execution, medication reminder, diet plan, exercise plan, cost management, and other functions.

3.4.4. System Support Management. The system provides a support interface to the BOSS system to synchronize user package ordering relationships and user service list records for the billing system to perform pricing, accounting, and settlement. For important service items, the system can remind medical staff and users of affairs, including notification of key process links, reminders of medication, and advance notice of affairs. Managers can issue announcements to users or set up various questionnaire reports and can view statistical information of user feedback results.
3.4.5. User Self-Service WEB Portal. The system provides users with a WEB self-service portal. The self-service functions include basic information management, pathological data viewing, medical advice and health information viewing, and expense list viewing. Users can apply for appointment registration, questionnaire feedback, and online message on the WEB portal. Users can view the current package information and package usage through the WEB portal. Users can leave messages online through WEB and consult health management related services online, and the agent is responsible for online answers.

\section{Results and Discussion}

4.1. System Performance Analysis. Part of the use cases of the remote medical platform performance test database is divided into 400, 500, 600, and 700 user statistical test cases according to the number of concurrent users. The test case of the database is obtained from EHR Medical History. The basic situation of the test is shown in Table 1 .

In the test results of this scenario, we can see that the CPU utilization is $40 \%$, the internal usage is $7.44 \mathrm{G}$, the memory utilization is $11.8 \%$, and the network bandwidth is $591.87 \mathrm{~m}$. During the whole test process, the indicators are stable, and there are no restart, crash, and other phenomena, so the system performance meets the design requirements. The CPU test data are shown in Table 2.

The average response time of the system and the processing time of concurrent users are shown in Figure 3. In the test results of this scenario, under the concurrency of $600 \mathrm{VU}$, the transaction processing speed reached 39.501 transactions per second, and the processing speed of failed transactions was 0.071 transactions per second, accounting for $0.18 \%$, which is less than the requirement of $5 \%$.

The system uses Bluetooth communication technology to collect the blood oxygen and pulse information collected in real time at the sensing layer through the intelligent terminal at the convergence layer and send it to the community health service center to realize real-time monitoring of personal blood oxygen and pulse information and medical data archiving. The judging basis for the blood pressure value in the system is shown in Table 3 .

The remote blood pressure measurement equipment is shown in Figure 4. The blood pressure list information includes blood pressure (high/low pressure), status, collection date, data source, and these four data records. The tester only needs to wear the blood pressure measurement device, and the blood pressure measurement device will read 
TABLE 1: Basic test situation.

\begin{tabular}{lc}
\hline Item & 600 simulate user concurrency and obtain user medical history data \\
\hline Use case overview & Add 20 users every 10 seconds \\
\hline Test steps & $\begin{array}{c}\text { Continue to pressurize for } 30 \text { minutes } \\
\text { Stop all users at the same time }\end{array}$ \\
\hline & Failure rate $<5 \%$ \\
Expected outcome & Average response time $\leq 20$ seconds \\
& Average CPU usage $\leq 80 \%$ \\
Memory usage $\leq 95 \%$
\end{tabular}

TABLe 2: CPU test data.

\begin{tabular}{lcccc}
\hline CPU test & Last $(\%)$ & Min (\%) & Avg (\%) & Max (\%) \\
\hline CPU system time & 3.79 & 3.79 & 4.45 & 4.54 \\
CPU user time & 424 & 42.1 & 43.27 & 47.76 \\
\hline
\end{tabular}

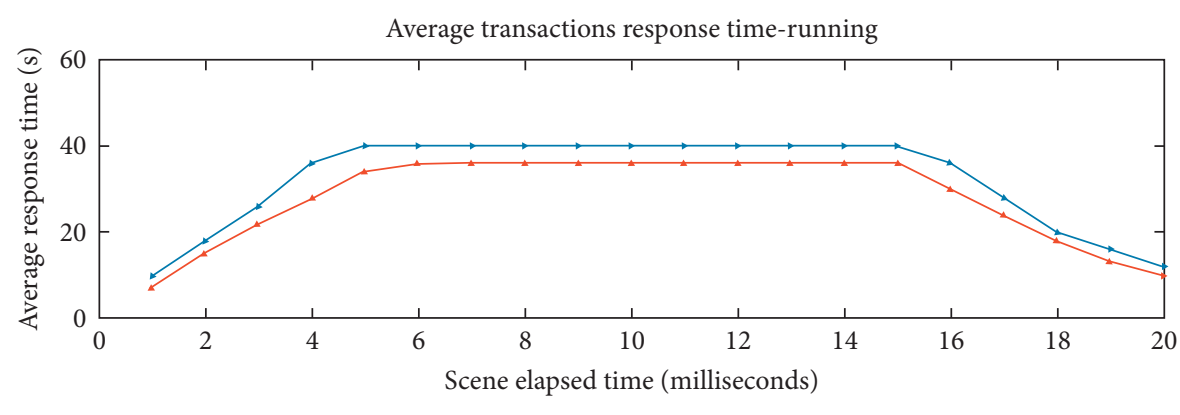

$\rightarrow$ Action transaction: pass

$\rightarrow$ App center: pass

(a)

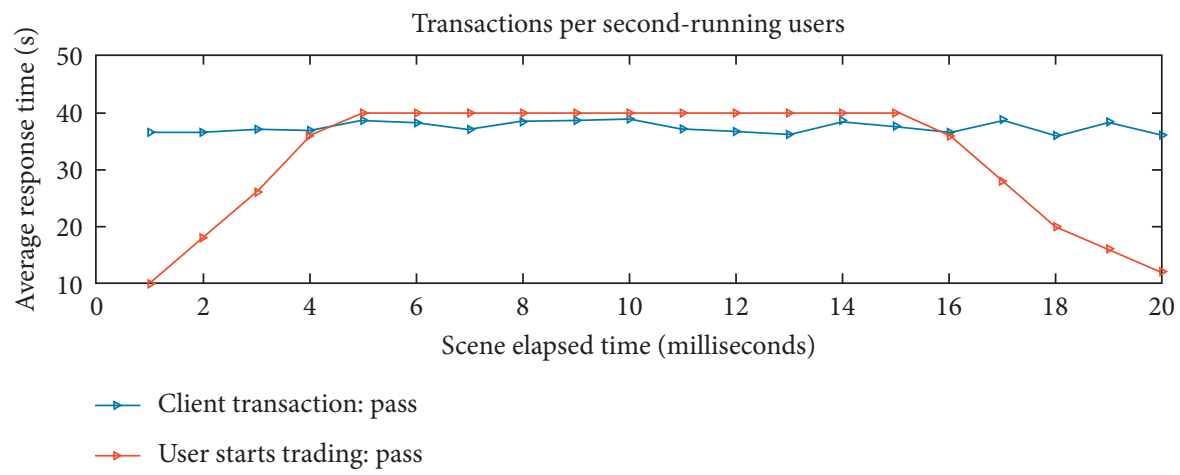

(b)

FIGURE 3: The average response time of the system and the processing time of the number of concurrent users.

TABLE 3: Judgment basis for the blood pressure value in the system.

\begin{tabular}{lcc}
\hline Classification & Systolic blood pressure $(\mathrm{mmHg})$ & Diastolic blood pressure $(\mathrm{mmHg})$ \\
\hline Normal blood pressure & $<130$ & $<85$ \\
Normal high blood pressure & $130-139$ & $85-89$ \\
Grade 1 hypertension & $140-159$ & $90-99$ \\
Grade 2 hypertension & $\geq 160$ & $\geq 100$ \\
\hline
\end{tabular}




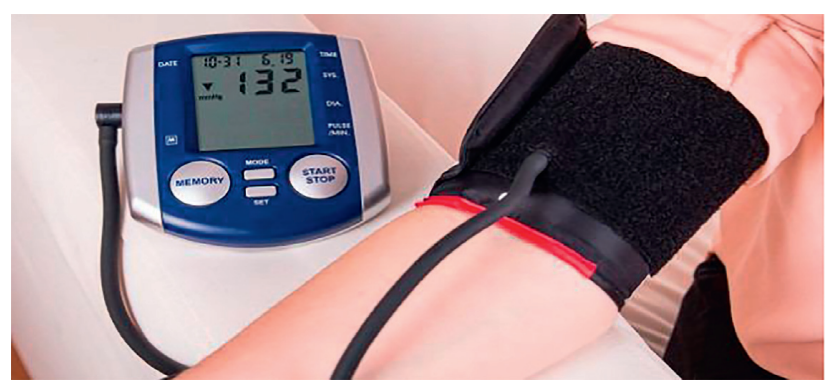

FIGURE 4: Remote blood pressure measurement equipment (http://alturl.com/fktvz).

TABLE 4: The main steps of the blood pressure measurement test on the mobile phone application platform.

\begin{tabular}{|c|c|}
\hline Step & Summary \\
\hline $\begin{array}{l}\text { Click "blood pressure measurement" in the } \mathrm{r} \\
\text { options list }\end{array}$ & $\begin{array}{c}\text { (Blood pressure measurement) verification click to enter the blood pre } \\
\text { measurement page }\end{array}$ \\
\hline Click on blood pressure measurement & $\begin{array}{c}\text { (Blood pressure measurement) verify that the sphygmomanometer is not } \\
\text { paired }\end{array}$ \\
\hline Click on blood pressure measu & $\begin{array}{c}\text { (Blood pressure measurement) verify that the sphygmomanometer has been } \\
\text { paired }\end{array}$ \\
\hline hygmomanometer page is found, click the "Retry" & $\begin{array}{l}\text { (Blood pressure measurement) verify that no blood pressure monitor page is } \\
\text { found }\end{array}$ \\
\hline $\begin{array}{l}\text { View the title display of the blood pressure measurement } \\
\text { page }\end{array}$ & (Blood pressure measurement) verify blood pressure measurement \\
\hline
\end{tabular}

the blood pressure measurement value and then automatically remotely transmit it to the receiving end.

The main steps of the blood pressure measurement test on the mobile phone application platform are shown in Table 4.

Use this system to measure blood pressure among randomly selected investigators, and the measurement results are shown in Figure 5. In the 20-day blood pressure measurement situation, the patient's blood pressure value is relatively stable.

4.2. System Application Analysis. 300 questionnaires were issued and 103 valid questionnaires were returned. The research conducted frequency analysis on the basic conditions of 103 valid questionnaires, and the specific results are shown in Table 5. In the survey sample, males and females accounted for $43.7 \%$ and $56.3 \%$ of the total survey samples, respectively, and the ratio of males to females was not much different. In terms of age group, mainly people over 75 years old and 65-69 years old account for $29.1 \%$ and $26.2 \%$ of the total sample population, respectively. People 60-64 years old and 70-74 years old both account for $16.5 \%$ of the total population, and people 55-59 years old are the least, accounting for $11.7 \%$ of the total population.

The medical needs survey is shown in Table 6. It can be seen from Table 6 that the average value of each dimension of medical service demand is in descending order: psychological comfort category, leisure and entertainment category, product problem-oriented category, and medical and health category. And the average value of psychological comfort services is 2.02, which is at the upper-middle level; the average values of leisure and entertainment services and product-oriented services are also at the upper-middle level, while the medical and health services are at the lower-middle level.

The telemedicine system's cardiac tests at different times are shown in Figure 6. Community service personnel can customize the content that users need to confirm and understand in consultation questions into a formatted form according to disease classification, so that residents can choose the corresponding form provided by their nanny to ask questions about their own health problems when asking questions about health consultation. The editing content of the customized form can be added in multiple ways such as single selection, multiple selection, text, and drop-down lists, which can realize diversified customization of the consultation form.

Community service personnel send health reminders through the station letter and can give advice and reminders on precautions, diet management, and disease management according to the corresponding diseases of the subdivided groups. Figure 7 shows the implementation of the special notification reminder interface that the service staff sends to a specific user to analyze the health data. Specific prompts are given through the examination of various indicators of the patient.

The importance of health monitoring products is shown in Figure 8. Regarding product problems, $72.8 \%$ of the population believe that it is important to design a product to provide elderly care services for the elderly; $56.3 \%$ of the population believe that it is important to wear health monitoring equipment, save health data in real time, and allow their children to see their health status every day; $50.5 \%$ of crowd believe that real-time positioning that allows your children to see your specific location is of general 


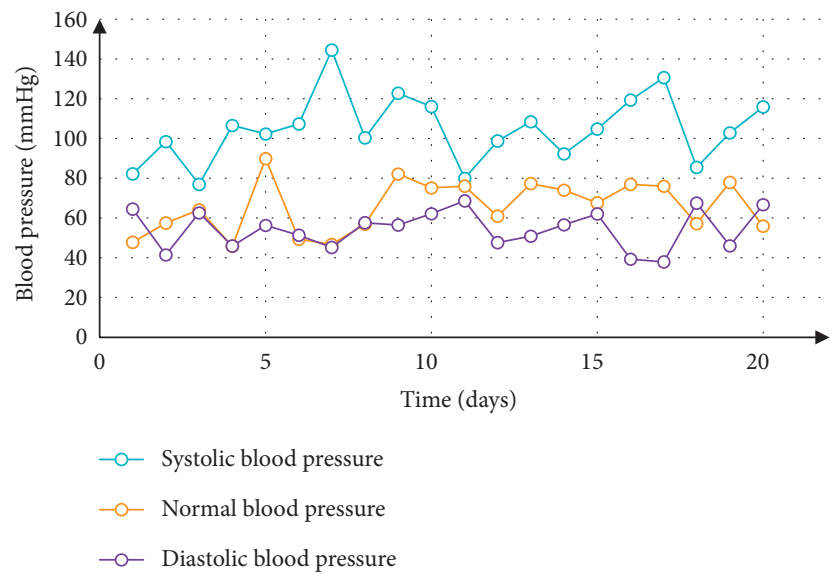

FIGURE 5: Using the system to measure blood pressure among randomly selected investigators.

TABLE 5: Basic situation of survey respondents.

\begin{tabular}{lcc}
\hline Specific performance & Number of people & Percentage \\
\hline Male & 45 & 43.7 \\
Female & 58 & 56.3 \\
$55-59$ years old & 12 & 11.7 \\
$60-64$ years old & 17 & 16.5 \\
$65-69$ years old & 27 & 26.2 \\
$70-74$ years old & 17 & 16.5 \\
Over 75 years old & 30 & 29.1 \\
\hline
\end{tabular}

TABLE 6: Medical needs survey.

\begin{tabular}{lccc}
\hline Types & Minimum & Maximum & Mean \\
\hline Medical and health & 1 & 2.3 & 1.30 \\
Leisure and entertainment & 1 & 3 & 1.73 \\
Psychological comfort & 1 & 3 & 2.02 \\
For product problems & 1 & 3 & 1.60 \\
\hline
\end{tabular}



FIgURE 6: Heart tests performed by the telemedicine system at different times (http://alturl.com/owc2y). 


\begin{tabular}{|c|c|c|c|c|c|c|c|}
\hline $\begin{array}{l}\text { Cardiovascular, } \\
\text { Drug }\end{array}$ & Target & Partner & Indication & P1 & $\mathrm{P} 2$ & P3 & $\mathrm{C}$ \\
\hline $\begin{array}{l}\text { IONIS-FXI } \\
(\text { BAY 2306001) }\end{array}$ & Factor XI & Bayer & Clotting disorders & & & & \\
\hline AKCEA-APO(A)- $\mathrm{L}_{\mathrm{RX}}$ & Apo(a) & Akcea/novartis & CVD & & & & \\
\hline AKCEA-APOCIII-L $\mathrm{L}_{\mathrm{RX}}$ & ApoCIII & Akcea/novartis & CVD & & & & \\
\hline IONIS-AGT-L $\mathrm{L}_{\mathrm{RX}}$ & AGT & - & $\begin{array}{l}\text { Treatment-resistant } \\
\text { hypertension }\end{array}$ & & & & \\
\hline
\end{tabular}

Figure 7: The implementation of the interface for the service personnel to send special notifications for health data analysis for a specific user (http://alturl.com/4rpyh).

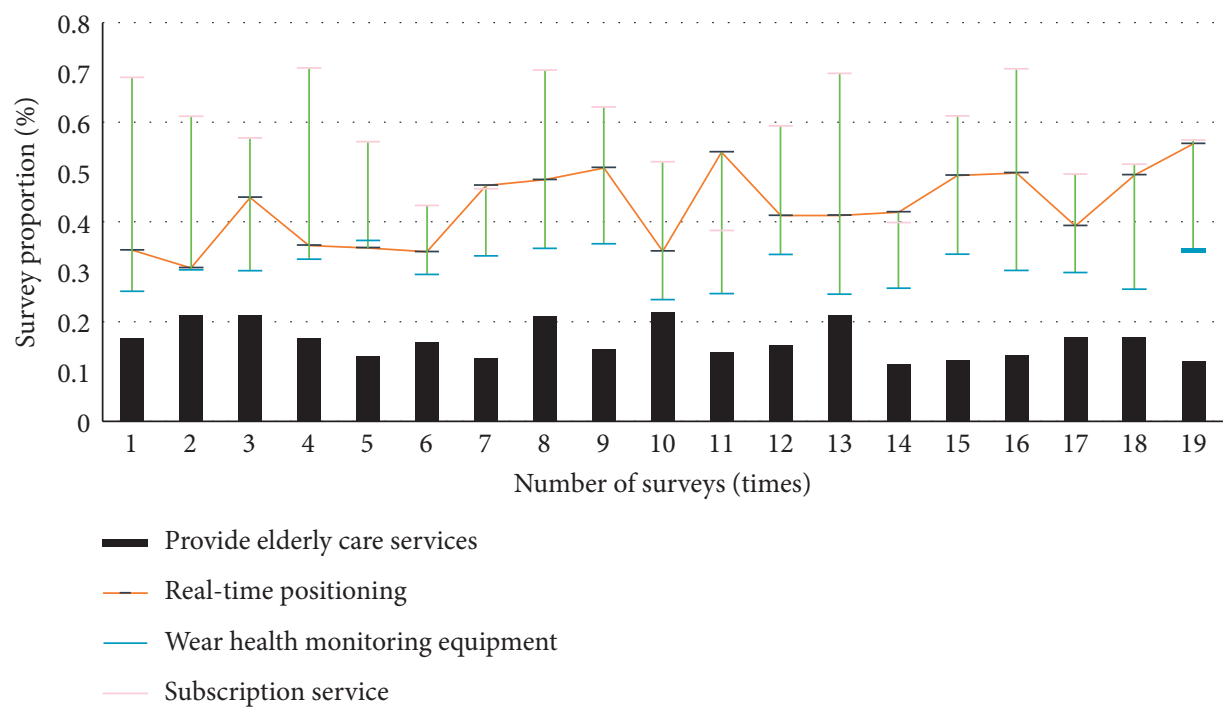

FIGURE 8: The importance of health monitoring products.

importance. $40.8 \%$ of the population believe that real-time positioning that allows your children to see the services of your specific location is very important. These data results show that most of the survey sample people think that it is important to design a product to provide elderly care services for the elders, wear health monitoring equipment, save health data in real time, and allow children to see their health status every day.

\section{Conclusion}

This research mainly discusses the design of medical and health monitoring IoT system based on cloud computing. There are three devices between users and health service providers: sensor terminals, gateway terminals, and business platforms. The sensor terminal is used to measure physiological indicators, such as blood pressure, electrocardiogram, blood oxygen saturation, heart rate, and other physiological indicators; the gateway terminal is used to link the sensor terminal to receive physiological indicators and forward them to the business platform; the gateway is also used to receive the health issued by the server information and other instructions. In the community service mode, users can be divided into groups according to communities and regions, and corresponding service doctors and agent customer service staff (nurses) can be assigned. Users can collect personal physiological indicators through medical terminals at home or outside. These indicators and information are transmitted to the backend health platform system via the mobile GSM-TD communication network. Users can also view their own historical health records in detail through the WEB/WAP website. The opinion of a health consultant: through the integration capabilities of the health cloud platform, relying on the interconnection with professional medical institutions HIS, LIS, and other information systems, joint operation of special value-added services, such as appointment registration, maternal and child healthcare, and medical information communication, allows users to subscribe to health value-added services and enjoy remote services and guidance from professional medical institutions. During the entire test process, the indicators were stable, and there were no restarts, crashes, etc., so the system performance met the design requirements. 


\section{Data Availability}

The data that support the findings of this study are available from the corresponding author upon reasonable request.

\section{Conflicts of Interest}

Shihua Cao received his Ph.D. degree from the Faculty of Information Technology of Macau University of Science and Technology, Macau. He is currently a professor and senior engineer in Qianjiang College of Hangzhou Normal University. He was a visiting professor in the University of North Texas from 2013 to 2014. His research areas are mainly in wireless sensor networks, energy harvesting, Internet of Things, and health informatics. He has published 70 research papers and 5 books, and he owns 20 Chinese and international patents. Xin Lin was born in Nov 1988. She received her master degree from Nursing College of Bengbu Medical University, she currently works in the Third People's Hospital of Bengbu with major scientific research experience of participating in one provincial scientific research project, presiding over and participating in two municipal scientific research projects, publishing more than 10 papers, including one SCI, and participating in the compilation of one nursing graduate textbook and one health education series of Bengbu Medical College. Keyong $\mathrm{Hu}$ is currently an associate professor of Electronic Information Engineering in Qianjiang College of Hangzhou Normal University. He has received Ph.D. degree in 2016 from Zhejiang University of Technology, Hangzhou, China, in mechatronic engineering. His research interests include artificial intelligence and new energy technology. Lidong Wang was born on December 4, 1982. She received her Ph.D. degree from the College of Computer Science and Technology, Zhejiang University. She is currently an associate professor at Hangzhou Normal University. Her current research interests include image processing, machine learning, and text mining. Wenjuan Li received a Ph.D. degree in 2012 from Zhejiang University, Hangzhou, China, in computer science. She is currently an associate professor in Hangzhou Normal University; she is also a postdoctor in Shanghai Tiao Tong University and a visiting scholar in CLOUDS Lab at University of Melbourne. Her research interests include cloud computing, social network, and trust. Mengxin Wang graduated from Anhui University of Chinese Medicine with a bachelor's degree; she is currently a graduate student majoring in nursing in Hangzhou Normal University and a trainee nurse in the Affiliated Hospital of Hangzhou Normal University. Her research areas are mainly in nursing informatics. She has published 1 conference paper and attended 3 academic conferences. Yuchao Le received a bachelor's degree in nursing from Qianjiang College of Hangzhou Normal University. He is currently a graduate student majoring in nursing in Hangzhou Normal University. His research areas are mainly in nursing informatics and health informatics. He has published 1 conference paper and attended 3 academic conferences.

\section{Acknowledgments}

This work was supported by the Joint Funds of the Zhejiang Provincial Natural Science Foundation of China (Grant nos. LHY21E090004 and LY19F020022) and Hangzhou Philosophy and Social Science Planning Base Project (no. 2018JD59).

\section{References}

[1] A. K. Mishra, A. K. Tripathy, D. Puthal, and L. T. Yang, "Analytical model for Sybil attack phases in internet of things," IEEE Internet of Things Journal, vol. 6, no. 1, pp. 379-387, 2019.

[2] A. Shifa, M. Asghar, and M. Fleury, "Multimedia security perspectives in IoT," Journal of Information Security Research, vol. 7, no. 4, pp. 150-159, 2016.

[3] C.-H. Chen, M.-Y. Lin, and C.-C. Liu, "Edge computing gateway of the industrial internet of things using multiple collaborative microcontrollers," IEEE Network, vol. 32, no. 1, pp. 24-32, 2018.

[4] X. Li, Y. Wang, and G. Liu, "Structured medical pathology data hiding information association mining algorithm based on optimized convolutional neural network," IEEE Access, vol. 8, no. 1, pp. 1443-1452, 2020.

[5] M. Centenaro, L. Vangelista, A. Zanella, and M. Zorzi, "Longrange communications in unlicensed bands: the rising stars in the IoT and smart city scenarios," IEEE Wireless Communications, vol. 23, no. 5, pp. 60-67, 2016.

[6] E. Ronen, A. Shamir, A.-O. Weingarten, and C. O"Flynn, "IoT goes nuclear: creating a zigbee chain reaction," IEEE Security \& Privacy, vol. 16, no. 1, pp. 54-62, 2018.

[7] P. Gope and T. Hwang, "BSN-care: a secure IoT-based modern healthcare system using body sensor network," IEEE Sensors Journal, vol. 16, no. 5, pp. 1368-1376, 2016.

[8] P. Schulz, M. Matthe, H. Klessig et al., "Latency critical IoT applications in 5G: perspective on the design of radio interface and network architecture," IEEE Communications Magazine, vol. 55, no. 2, pp. 70-78, 2017.

[9] M. Shirvanimoghaddam, M. Dohler, and S. J. Johnson, "Massive non-orthogonal multiple access for cellular IoT: potentials and limitations," IEEE Communications Magazine, vol. 55, no. 9, pp. 55-61, 2017.

[10] J. Gozalvez, "New 3GPP standard for IoT [mobile radio]," IEEE Vehicular Technology Magazine, vol. 11, no. 1, pp. 14-20, 2016.

[11] T. Qu, S. P. Lei, Z. Z. Wang et al., "IoT-based real-time production logistics synchronization system under smart cloud manufacturing," International Journal of Advanced Manufacturing Technology, vol. 84, no. 1-4, pp. 147-164, 2016.

[12] A. Brogi and S. Forti, "QoS-aware deployment of IoT applications through the fog," IEEE Internet of Things Journal, vol. 4, no. 5, pp. 1185-1192, 2017.

[13] K. Lin, M. Chen, J. Deng, M. M. Hassan, and G. Fortino, "Enhanced fingerprinting and trajectory prediction for IoT localization in smart buildings," IEEE Transactions on Automation Science and Engineering, vol. 13, no. 3, pp. 12941307, 2016.

[14] C. Kolias, G. Kambourakis, A. Stavrou, and J. Voas, "DDoS in the IoT: Mirai and other botnets," Computer, vol. 50, no. 7, pp. 80-84, 2017.

[15] M. Chiang and T. Zhang, "Fog and IoT: an overview of research opportunities," IEEE Internet of Things Journal, vol. 3, no. 6, pp. 854-864, 2017. 
[16] R. S. Sinha, Y. Wei, and S.-H. Hwang, "A survey on LPWA technology: LoRa and NB-IoT,” Ict Express, vol. 3, no. 1, pp. 14-21, 2017.

[17] J. Duan, D. Gao, D. Yang et al., "An energy-aware trust derivation scheme with game theoretic approach in wireless sensor networks for IoT applications," IEEE Internet of Things Journal, vol. 1, no. 1, pp. 58-69, 2017.

[18] H. Li, K. Ota, and M. Dong, "Learning IoT in edge: deep learning for the internet of things with edge computing," IEEE Network, vol. 32, no. 1, pp. 96-101, 2018.

[19] D. Minoli, K. Sohraby, and B. Occhiogrosso, "IoT considerations, requirements, and architectures for smart buildingsenergy optimization and next-generation building management systems," IEEE Internet of Things Journal, vol. 4, no. 1, pp. 269-283, 2017.

[20] A. Broring, S. Schmid, C.-K. Schindhelm et al., "Enabling IoT ecosystems through platform interoperability," IEEE Software, vol. 34, no. 1, pp. 54-61, 2017.

[21] H. Cai, B. Xu, L. Jiang, and A. V. Vasilakos, "IoT-based big data storage systems in cloud computing: perspectives and challenges," IEEE Internet of Things Journal, vol. 4, no. 1, pp. 75-87, 2017.

[22] L. Kong, M. K. Khan, F. Wu, G. Chen, and P. Zeng, "Millimeter-wave wireless communications for IoT-cloud supported autonomous vehicles: overview, design, and challenges," IEEE Communications Magazine, vol. 55, no. 1, pp. 62-68, 2017.

[23] S. Alletto, R. Cucchiara, G. Del Fiore et al., "An indoor location-aware system for an IoT-based smart museum," IEEE Internet of Things Journal, vol. 3, no. 2, pp. 244-253, 2016.

[24] M. Elhoseny, G.-B. Bian, S. K. Lakshmanaprabu, K. Shankar, A. K. Singh, and W. Wu, "Effective features to classify ovarian cancer data in internet of medical things," Computer Networks, vol. 159, pp. 147-156, 2019.

[25] T. Song, R. Li, B. Mei, J. Yu, X. Xing, and X. Cheng, “A privacy preserving communication protocol for IoT applications in smart homes," IEEE Internet of Things Journal, vol. 4, no. 6, pp. 1844-1852, 2017.

[26] S. Wan, Z. Gu, and Q. Ni, "Cognitive computing and wireless communications on the edge for healthcare service robots," Computer Communications, vol. 149, 2019.

[27] A. Tewari and B. B. Gupta, "Cryptanalysis of a novel ultralightweight mutual authentication protocol for IoT devices using RFID tags," Journal of Supercomputing, vol. 73, no. 3, pp. 1-18, 2017.

[28] C. Perera, D. S. Talagala, C. H. Liu et al., "Energy-efficient location and activity-aware on-demand mobile distributed sensing platform for sensing as a service in IoT clouds," IEEE Transactions on Computational Social Systems, vol. 2, no. 4, pp. 171-181, 2016.

[29] M. Gharbieh, H. Elsawy, A. Bader et al., "Spatiotemporal stochastic modeling of IoT enabled cellular networks: scalability and stability analysis," IEEE Transactions on Communications, vol. 65, no. 8, pp. 3585-3600, 2017.

[30] J. Zhou, Z. Cao, X. Dong, and A. V. Vasilakos, "Security and privacy for cloud-based IoT: challenges," IEEE Communications Magazine, vol. 55, no. 1, pp. 26-33, 2017.

[31] Y. Jiang, H. Song, R. Wang, M. Gu, J. Sun, and L. Sha, "Datacentered runtime verification of wireless medical cyberphysical system," IEEE Transactions on Industrial Informatics, vol. 13, no. 4, pp. 1900-1909, 2017. 